\title{
Levy's stable distribution for stock crash detecting
}

\author{
Andrii Bielinskyi ${ }^{1, *}$, Serhiy Semerikov ${ }^{1}$, Viktoria Solovieva ${ }^{2}$, and Vladimir Soloviev ${ }^{1}$ \\ ${ }^{1}$ Kryvyi Rih State Pedagogical University, 54, Gagarina Ave, Kryvyi Rih, 50086, Ukraine \\ ${ }^{2}$ Kryvyi Rih Economic Institute of Kyiv National Economic University named after Vadym Hetman, 16, Medychna St., Kryvyi Rih, \\ 50000, Ukraine
}

\begin{abstract}
In this paper we study the possibility of construction indicators-precursors relying on one of the most power-law tailed distributions - Levy's stable distribution. Here, we apply Levy's parameters for 29 stock indices for the period from 1 March 2000 to 28 March 2019 daily values and show their effectiveness as indicators of crisis states on the example of Dow Jones Industrial Average index for the period from 2 January 1920 to 2019. In spite of popularity of the Gaussian distribution in financial modeling, we demonstrated that Levy's stable distribution is more suitable due to its theoretical reasons and analysis results. And finally, we conclude that stability $\alpha$ and skewness $\beta$ parameters of Levy's stable distribution which demonstrate characteristic behavior for crash and critical states, can serve as an indicator-precursors of unstable states.
\end{abstract}

\section{Introduction}

The efficient financial market is an integral part of the modern market economy. With a rapidly growing financial market, new risk management methods are becoming more demanded that take into account new non-Gaussian distributions. The task of monitoring and predicting of possible critical states of financial and economics systems are very relevant today. In our opinion, the availability of the time series for stock markets gives the opportunity to solve such tasks in very effective ways. Financial crises that regularly shake the world economy are characterized by noticeable fluctuations in stock indices, thereby causing noticeable changes in the statistical distributions of empirical data $[1,2]$. Consequently, the analysis of the form and parameters of the distribution of price fluctuations of the stock market indexes will make it possible to predict the possible occurrence of the financial crisis.

In 1900, Bachelier proposed the first model for the stochastic process of returns - an uncorrelated random walk with independent, identically Gaussian distributed (i.i.d) random variables [3]. This model is natural if one considers the return over a time scale $\Delta \mathrm{t}$ to be the result of many independent "shocks", which then lead by the central limit theorem to a Gaussian distribution of returns [3]. However, empirical studies [4-6] show that the distribution of returns has pronounced tails in striking contrast to that of a Gaussian.

For time series $S(t)$ which describes the dynamics of price on stock index, the returns $g(t)$ over some time scale $\Delta t$ is defined as the forward changes in the logarithm of $S(t)$,

$$
g(t) \equiv(\ln S(t+\Delta t) / \ln S(t)) .
$$

For small changes in the price, the returns $g(t)$ is approximately the forward relative change

$$
g(t) \approx \frac{S(t+\Delta t)-S(t)}{S(t)} .
$$

To illustrate mentioned above fact, we show in Fig. 1 the daily returns of the DJIA market index for 19002019 and contrast it with a sequence of i.i.d. Gaussian random variables.

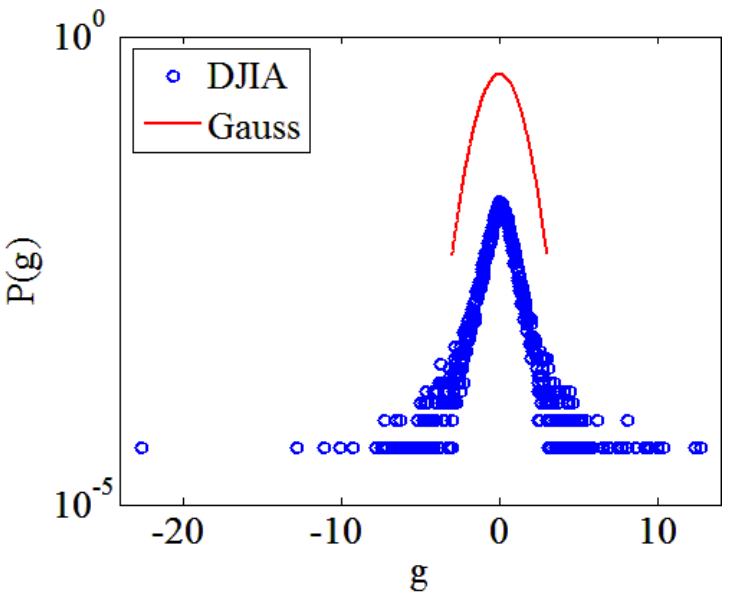

Fig. 1. Probability density function of DJIA daily normalized returns during the period from 1900 to 2019.

It is obvious that the distribution of returns has heavy tails and in the general case can be described as

$$
P(g>x) \sim x^{-(1+\alpha)}, \quad \alpha \in(0,2]
$$


and for stock indexes it has a universal look, known as the cubic laws of stock market activity [6].

Fig. 2 confirms the cubic law for the DJIA index.

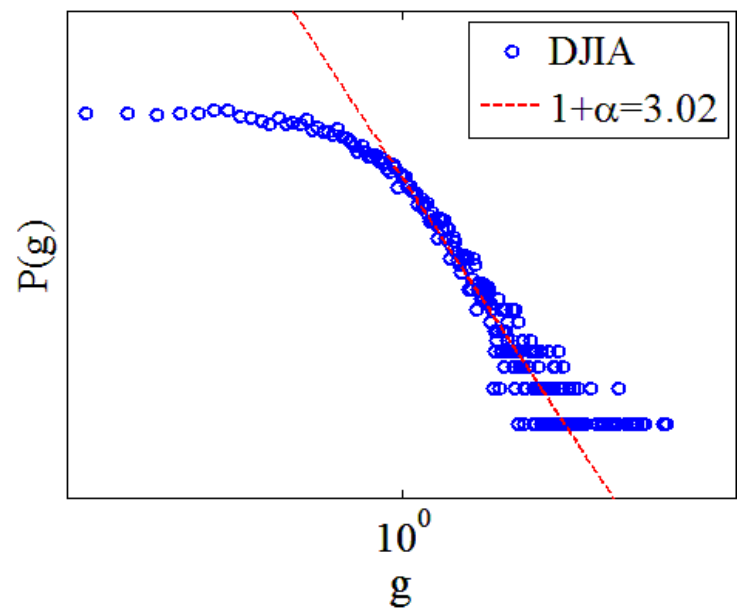

Fig. 2. Cumulative distributions of the normalized DJIA daily returns. Fits yield values $\alpha=2.02 \pm 0.02$.

In the analysis of cotton prices, Mandelbrot observed that in addition to being non-Gaussian, the process of returns shows another interesting property: "time scaling" - that is, the distributions of returns for various choices of $\Delta t$, ranging from 1 day up to 1 month have similar functional forms [7]. Motivated by (i) pronounced tails, and (ii) a stable functional form for different time scales, Mandelbrot [7] proposed that the distribution of returns is consistent with a Levy stable distribution [8] - that is, the returns can be modeled as a Levy stable process. Levy stable distributions arise from the generalization of the Central Limit Theorem (CLT) to random variables which do not have a finite second moment.

The CLT [9], which offers the fundamental justification for approximate normality, points to the importance of $\alpha$-stable distribution: they are the only limiting laws of normalized sums of independent, identically distributed random variables. Gaussian distributions, the best known member of the stable family, have long been well understood and widely used in all sorts of problems. However, they do not allow for large fluctuations and are thus inadequate for modeling high variability. Non-Gaussian stable models, on other hand, do not share such limitations. In general, the upper and lower tails of their distributions decreases like a power function. In literature, this is often characterized as heavy or long tails. In the last two or three decades, data which seem to fit the stable model has been collected in fields as diverse as economics, telecommunications, hydrology and physics (see for example [6]).

During our research of Levy's stable distribution, applied for the stock market, we have found that there are many articles, which were devoted to it [4-6, 10-12]. Consequently, it was pointed out that Levy's stable distribution fits better that the Gaussian distribution to financial markets. It is still debatable whether Levy's stable distribution is appreciable, since there is not enough theoretical material and there is not a universal analyzing method for estimating parameters of Levy's stable distribution.

Therefore, during our research we discuss theoretical material applied to Levy's stable distribution, and discuss whether it acceptable for indicating crisis states on financial markets or not.

Our research structured as follows. Section 2 is introduction to Levy's stable distribution and its properties. Section 3 describes different approaches for estimating stable distribution parameters. In Section 4 we described how to estimate Levy's stable distribution and which method the most appreciable method for calculating its parameters. Section 5 present classified DJIA price data, and obtained results.

\section{Levy's stable distribution properties}

Levy's stable distribution being the generalization of the CLT, became an addition to a wide class of distributions. Assume that $P_{n} \equiv \sum_{i=1}^{n} x_{i}$ is the sum of i.i.d. random variables $x_{i}$. Then, if the variables $x_{i}$ have finite second moment, the CLT holds and $P_{n}$ is distributed as a Gaussian in the limit $n \rightarrow \infty$.

In case when the random variables $x_{i}$ are characterized by a distribution having asymptotic powerlaw behavior (3) $P_{n}$ will converge to a Levy stable stochastic process of index $\alpha$ in the limit $n \rightarrow \infty$.

Stable distribution is presented by 4 parameters: $\alpha \in(0,2]$ is the stability parameter, $\beta \in[-1,1]$ the skewness parameter, $\gamma \in[0, \infty)$ the scale parameter and $\delta \in(-\infty, \infty)$ the location parameter. Since the variables $x_{i}$ is characterized by four parameters, we will denote $\alpha$ stable distribution by $S(\alpha, \beta, \gamma, \delta)$ and write

$$
x \sim S(\alpha, \beta, \gamma, \delta)
$$

Stable distribution has a property that the mean cannot be defined for $\alpha \in(0,1]$ and the variance diverges for $\alpha \in(0,2)$.

Furthermore, the Levy stable distributions cannot be defined in closed form for a few cases: the case of $(\alpha, \beta)=(2,0)$ corresponds to the Gaussian distribution, $(\alpha, \beta)=(1,0)$ to the Cauchy distribution. Instead, it is expressed in terms of their Fourier transforms or characteristic functions (CF), which we denote as $\lambda(k)$, where $k$ denotes the Fourier transformed variable.

For Levy stable distribution, if the variable $x_{i}$ follows $S(\alpha, \beta, \gamma, \delta)$, the CF can be expressed as [13]

$$
\lambda(k)=\left\{\begin{array}{ll}
\exp \left\{i \delta k-|\gamma k|^{\alpha}\left[1+i \beta \frac{k}{|k|} \operatorname{tg}\left(\frac{\pi \alpha}{2}\right)\right]\right\}, & (\alpha \neq 1) \\
\exp \left\{i \delta k-|\gamma k|^{\alpha}\left[1+i \beta \frac{k}{|k|} \ln |k|\right]\right\}, & (\alpha=1)
\end{array} .\right.
$$

It worth considering that with value of $\beta=0$, the distribution is symmetric, right-tailed if positive, and left-tailed if negative. 


\section{Methods for estimation of stable law parameters}

There are numerous approaches which can estimate stable distribution parameters. Since the probability density functions is not always expressed in a closed form, there are some challenges to overcome the analytic difficulties. Thus, there have been constructed a variety of methods: the approximate maximum likelihood estimation $[14,15]$, quantiles method $[16,17]$, fractional lower order moment method [18, 19], method of logcumulant [20], the logarithmic moment method [21] and more. Unfortunately, some of those methods cannot be applied due to computational problems associated with limited range of estimation, restricted range of parameters, high computational costs, or requiring large number of data. However, several of them should be mentioned.

\subsection{Maximum Likelihood Method}

DuMouchel was the first to obtain approximate ML estimates of $\alpha$ and $\gamma$ (assuming $\delta=0$ ) [22]. A multinomial approximation to the likelihood function is used in his approach. Under some additional assumptions on $\hat{\alpha}$ and the likelihood function, DuMouchel has shown the obtained estimates to be consistent and asymptotically normal. However, the computational effort involved seems considerable.

A direct method can be formulated, after Brorsen and Yang [14], as follows. The standard symmetric probability density functions defined by Zolotarev [23] is presented as

$$
f_{\alpha}(x)=\frac{\alpha}{\pi|1-\alpha|} x^{1 /(\alpha-1)} \int_{0}^{\pi / 2} U_{\alpha}(\eta, 0) e^{-x^{\alpha /(\alpha-1) U_{\alpha}(\eta, 0)}} d \eta,
$$

for $\alpha \neq 1, x>0$, where $U_{\alpha}$ is defined by

$$
U_{\alpha}\left(\eta, \eta_{0}\right)=\left(\frac{\sin \alpha\left(\eta-\eta_{0}\right)}{\cos \eta}\right)^{\alpha /(1-\alpha)} \frac{\cos \left(\eta-\alpha\left(\eta-\eta_{0}\right)\right)}{\cos \eta},
$$

and $\eta_{0}$ is explained here [24]. Therefore, the parameters $\alpha, \gamma$ and $\delta$ can be estimated from the observations $x_{i}$ $(i=1,2, \ldots, n)$ by maximizing the log likelihood function

$$
\begin{gathered}
\sum_{i=1}^{n} \log f_{\alpha}\left(z_{i}\right)=n \log \alpha-n \log (\alpha-1) \pi+\sum_{i=1}^{n} \frac{\log z_{i}}{\alpha-1}+ \\
+\sum_{i=1}^{n} \log \int_{0}^{\pi / 2} U_{\alpha}(\eta, 0) e^{-z_{i}^{\alpha /(\alpha-1)} U_{\alpha}(\eta, 0)} d \eta
\end{gathered}
$$

where $z_{i}=\left|x_{i}-\delta\right| / \gamma$.

To avoid the discontinuity and nondifferentiability of the symmetric $\alpha$-stable density function at $\alpha=1, \alpha$ is restricted to be greater than one. Caution must be used when evaluating the integrals (6) and (8), since the integrals are singular at $\eta=0$.
An obvious disadvantage of this method is that it is a highly nonlinear optimization problem and no initialization and convergence analysis is available.

\subsection{Sample Quantiles Methods}

Let $x_{i}$ be the $f$-th population quantile, so that $S(\alpha, \beta, \gamma, \delta)\left(x_{i}\right)=f$. Let $\hat{x}_{f}$ be the corresponding sample quantile, i.e. $\hat{x}_{f}$ satisfies $F_{n}\left(\hat{x}_{f}\right)=f$. As McCulloch [17] points out, to avoid spurious skewness in finite samples, a correction must be made. If the $x_{i}$ 's are arranged in ascending order, the correction may be performed by identifying $x_{i}$ with $\hat{x}_{q(i)}$, where $q(i)=\frac{2 i-1}{2 n}$, and then interpolating linearly to $f$ from the two adjacent $q(i)$ values. Then $\hat{x}_{f}$ is a consistent estimator of $x_{f}$, the $f$ quantile.

\subsection{Regression Method}

Koutrouvelis [13, 25] presented a regression type method of estimating the four parameters of stable distribution. It is based on the following algorithm concerning the CF. From (5) it can be derived that

$$
\log \left(-\log |\lambda(k)|^{2}\right)=\log \left(2 \gamma^{\alpha}\right)+\alpha \log |k|
$$

The real and imaginary parts of $\lambda(k)$ are for $\alpha \neq 1$ given by

$$
\lambda_{\mathrm{R}}(k)=\exp \left(-|\gamma k|^{\alpha}\right) \cos \left[\delta k+|\gamma k|^{\alpha} \beta \operatorname{sign}(t) \operatorname{tg}\left(\frac{\pi \alpha}{2}\right)\right],
$$

and

$$
\lambda_{\mathrm{I}}(k)=\exp \left(-|\gamma k|^{\alpha}\right) \sin \left[\delta k+|\gamma k|^{\alpha} \beta \operatorname{sign}(t) \operatorname{tg}\left(\frac{\pi \alpha}{2}\right)\right] .
$$

The last two equations lead, apart from considerations of principal values, to

$$
\operatorname{arctg}\left(\frac{\lambda_{\mathrm{I}}(k)}{\lambda_{\mathrm{R}}(k)}\right)=\delta k+\beta \gamma^{\alpha} \operatorname{tg}\left(\frac{\pi \alpha}{2}\right) \operatorname{sign}(k)|k|^{\alpha} .
$$

Equation (9) depends only on $\alpha$ and $\gamma$ and suggests that we estimate these parameters by regressing

$$
y=\log \left(-\log \left|\lambda_{N}(k)\right|^{2}\right)
$$

on $\omega=\log |k|$ in the model

$$
y_{n}=m+\alpha \omega_{n}+\varepsilon_{n}, n=1,2, \ldots, N,
$$

where $\left(k_{n}\right)$ is an appropriate set of real numbers, $m=\log \left(2 \gamma^{\alpha}\right)$, and $\varepsilon_{n}$ responds for an error term.

With estimated and fixed parameters $\alpha$ and $\gamma$, the values of $\beta$ and $\delta$ can be obtained by using equation (9). Let $g_{n}(u)=\operatorname{Arctg}\left(\lambda_{\mathrm{I}, n} / \lambda_{\mathrm{R}, n}\right)$, where $\operatorname{Arctg}$ denotes the principal value of the arctan function. Then we can 
estimate $\beta$ and $\delta$ by regressing $z=g_{n}(u)+\pi \tau_{n}(u)$ on $u$ and $\operatorname{sign}(u)|u|^{\alpha}$ in the model

$$
z_{l}=\delta u_{l}+\beta \gamma^{\alpha} \operatorname{tg}\left(\frac{\pi \alpha}{2}\right) \operatorname{sign}\left(u_{l}\right)\left|u_{l}\right|^{\alpha}+v_{l}, l=1, \ldots, L,
$$

where $\left(u_{l}\right)$ is an appropriate set of real numbers and $v_{l}$ denotes an error term.

As it was mentioned before, most of these methods have high computational costs, restricted ranges of parameters or require a large number of data. Thus, we would like to use simple approach proposed by Koutrovelis [25] which is based on CF and it is tested to be valid and clears the above issues.

\section{Estimation of Levy's stable distribution}

When we analyze data, we often assume that they are ergodic [26]. In general, if random variables $x_{n}(n=1,2$, $\ldots, N)$ are ergodic with the integrable function $f(x)$, the preserving map $T(x)$ and the measure $p(x) d x$ in the space $M$, then the following equation holds [27]:

$$
\lim _{N \rightarrow \infty} \frac{1}{N} \sum_{n=1}^{N} f\left(T^{n} x\right)=\int_{M} f(x) p(x) d x .
$$

Then, to consider characteristic functions, equation (13) comes out to be the following ergodic equality [27]:

$$
\lim _{N \rightarrow \infty} \frac{1}{N} \sum_{n=1}^{N} \exp \left(i k x_{i}\right)=\int_{-\infty}^{\infty} \exp (i k x) f(x) d x,
$$

for which we have

$$
\hat{\lambda}(k)=\lim _{N \rightarrow \infty} \frac{1}{N} \sum_{n=1}^{N} \exp \left(i k x_{i}\right) .
$$

This assumption allows us to empirically obtain the probability distribution. Hence, the empirical characteristic function $\lambda_{N}(k)$ of a large number of data set $x_{n}(n=1,2, \ldots, N)$ can be calculated as

$$
\hat{\lambda}_{N}(k)=\frac{1}{N} \sum_{n=1}^{N} \exp \left(i k X_{n}\right) .
$$

When the data follow Levy's stable distribution with the parameters $(\alpha, \beta, \gamma, \delta)(\alpha \neq 1, k>0)$, the characteristic function can be presented as

$$
\hat{\lambda}_{N}(k)=\exp \left[i \delta k-(\gamma k)^{\alpha}\left\{1+i \beta \tan \left(\frac{\pi \alpha}{2}\right)\right\}\right]
$$

from equation (5). With equation (17), we can derive

$$
\log \left(-\log \left|\hat{\lambda}_{N}(k)\right|\right)=\hat{\alpha} \log k+\hat{\alpha} \log \hat{\gamma}
$$

and

$$
\frac{1}{k} \operatorname{arctg}\left\{\frac{\hat{\lambda}_{N, \mathrm{I}}(k)}{\hat{\lambda}_{N, \mathrm{R}}(k)}\right\}=-\hat{\beta} \hat{\gamma}^{\hat{\alpha}} \operatorname{tg}\left(\frac{\pi \hat{\alpha}}{2}\right) k^{\hat{\alpha}-1}+\hat{\delta},
$$

where each of $\hat{\lambda}_{N, \mathrm{I}}(k)$ and $\hat{\lambda}_{N, \mathrm{R}}(k)$ corresponds to the imaginary and real part of the empirical CF. Through linear regression method in equations (18) and (19) around $k=0$ the parameters $(\alpha, \beta, \gamma, \delta)$ can be estimated. In case when $(\gamma, \delta)$ are far from the standard value of $(1,0)$, each parameter can not be estimated accurately. In this case the data should be normalized to $(\gamma, \delta)=(1,0)$ and then $(\alpha, \beta)$ can be estimated.

While the standard estimation method use the probability density function from the actual data with difficulty in estimating the tails of the distribution which are essentially important part of Levy's stable distribution, the method which we use in this paper can indicate the tail through the characteristic function. In addition, this method has a faster convergence according to the increasing number of data. The introduced integer $\tau_{n}(u)$ accounts for possible nonprincipal branches of the arctan function.

\section{Data classification of Dow Jones Industrial Average}

In this paper we have estimated Levy's parameters for stock indices for the period from 1 March 2000 to 28 March 2019 daily values. This data include stock indices of developed countries, developing and emergent markets. The data were downloaded from Yahoo Finance (http://finance.yahoo.com) and Investing.com (https://www.investing.com). The distribution parameters were found for the entire time series and the algorithm of a moving window. For moving window, the part of the time series (window), for which there were calculated corresponding parameters, was selected. Then, the window was displaced along the time series in definite increment (step) and the procedure repeated until all the studied series had exhausted. For our case the window width is 500 and 1000 days, time step 1 and 5 days. The calculation results for the whole time series of the order-decreasing parameter $\alpha$ are shown in Table 1.

The considered stock indices for the specified period include crisis phenomena and these periods obviously affect the dynamics of distribution parameters. Therefore, you should calculate them in the model of the moving window and compare their dynamics with the dynamics of the original time series.

For analysing and explaining basic characteristics of complex systems with $\alpha$-stable distribution, we have chosen Dow Jones Industrial Average index (DJIA) as the most quoted financial barometer in the world. In addition, like complex systems, financial markets fascinating examples of complexity: a real world complex system whose evolution is dictated by the decisions of many people, generating huge amounts of data. For understanding of the falls that occurred on this market, we analysed different scientific articles [31-32], 
and relying on our research, we classified them on crashes and critical event, and separated DJIA time series into two parts where first part occupies period from 2 January 1920 to 3 January 1983 and second part from 4 January 1983 to 18 March 2019, for having better overview of its dynamics. Note that the data set here is an every 1-day data, except those when stock market closed and does not work. During our research it was established that:

1. Crashes are short, time-localized drops, with strong losing of price each day.

2. Critical events are those falls that, during their existence, have not had such serious changes in price as crashes.

Table 1. The calculated Levy's stable parameters $\alpha$ and $\beta$ for the considered stock indices. The results were obtained for the length of window 500 and time step 1 day.

\begin{tabular}{|l|l|c|c|}
\hline $\mathrm{N}$ & \multicolumn{1}{|c|}{ Index } & $\alpha$ & $\beta$ \\
\hline 1 & Nikkei 225 & 1.71 & -0.240 \\
\hline 2 & IBEX 35 & 1.71 & -0.206 \\
\hline 3 & CAC 40 & 1.70 & -0.247 \\
\hline 4 & OMX Stockholm 30 & 1.70 & -0.152 \\
\hline 5 & FTSE MIB & 1.69 & -0.374 \\
\hline 6 & Swiss Market Index & 1.69 & -0.210 \\
\hline 7 & DAX PERFORMANCE & 1.68 & -0.199 \\
\hline 8 & FTSE 100 & 1.68 & -0.180 \\
\hline 9 & Warsaw Stock Exhange WIG & 1.68 & -0.022 \\
\hline 10 & BEL 20 & 1.67 & -0.236 \\
\hline 11 & TA 35 & 1.67 & -0.186 \\
\hline 12 & KOSPI Composite & 1.66 & -0.304 \\
\hline 13 & S\&P/TSX Composite & 1.60 & -0.349 \\
\hline 14 & AEX & 1.60 & -0.214 \\
\hline 15 & BIST 100 & 1.60 & -0.120 \\
\hline 16 & Dow Jones Industrial Average & 1.59 & -0.126 \\
\hline 17 & BOVESPA & 1.58 & -0.080 \\
\hline 18 & Hang Seng & 1.58 & -0.153 \\
\hline 19 & S\&P 500 & 1.57 & -0.151 \\
\hline 20 & IPC MEXICO & 1.48 & -0.118 \\
\hline 21 & NASDAQ Composite Index & 1.48 & -0.139 \\
\hline 22 & RTS Index & 1.46 & -0.081 \\
\hline 23 & BSE Sensex 30 & 1.44 & -0.027 \\
\hline 24 & Nifty 50 & 1.42 & -0.047 \\
\hline 25 & Jakarta Stock Exchange Composite & 1.27 & -0.043 \\
\hline 26 & Shanghai Composite & 1.27 & -0.046 \\
\hline 27 & KSE 100 & 1.05 & -0.050 \\
\hline 28 & Ukraine PFTS & 0.83 & -0.089 \\
\hline 29 & S\&P Merval & 0.74 & -0.055 \\
\hline
\end{tabular}

As it is seen from the Table 1, during DJIA existence, many crashes and critical events shook it. According to our classification, events with number (1, $10,13,15)$ are crashes, all the rest - critical events. From the data above, we estimate the parameters $\alpha$ and $\beta$ of the stable distribution that the best describes the empirical returns.

Further, comparing the dynamics of the actual time series and the corresponding measures of complexity, we can judge the characteristic changes in the dynamics of the behavior of complexity with changes in the stock index. If the estimated parameter behaves in a definite way for all periods of crashes, for example, decreases or increases during the pre-critical period, then it can serve as an indicator-precursor of such a crashes phenomenon.

Table 2 shows the major crashes and critical events related to our classification.

Table 2. Major Historical Corrections since 1920.

\begin{tabular}{|c|c|c|c|}
\hline $\mathrm{N}$ & Interval & Days in correction & Decline, $\%$ \\
\hline 1 & $03.09 .1929-29.10 .1929$ & 41 & 39,64 \\
\hline 2 & $01.03 .1938-31.03 .1938$ & 23 & 24,15 \\
\hline 3 & $08.04 .1940-05.06 .1940$ & 42 & 25,10 \\
\hline 4 & $21.08 .1946-10.09 .1946$ & 14 & 16,35 \\
\hline 5 & $30.07 .1957-22.10 .1957$ & 60 & 17,51 \\
\hline 6 & $19.03 .1962-28.05 .1962$ & 50 & 19,91 \\
\hline 7 & $18.07 .1966-07.10 .1966$ & 59 & 12,84 \\
\hline 8 & $09.04 .1970-26.05 .1970$ & 34 & 20,35 \\
\hline 9 & $24.10 .1974-04.10 .1974$ & 52 & 27,45 \\
\hline 10 & $02.10 .1987-19.10 .1987$ & 12 & 34,16 \\
\hline 11 & $17.07 .1990-23.08 .1990$ & 28 & 17,21 \\
\hline 12 & $01.10 .1997-21.10 .1997$ & 15 & 12,43 \\
\hline 13 & $17.08 .1998-31.08 .1998$ & 11 & 18,44 \\
\hline 14 & $14.08 .2002-01.10 .2002$ & 34 & 19,52 \\
\hline 15 & $16.10 .2008-15.12 .2008$ & 42 & 30,21 \\
\hline 16 & $09.08 .2011-22.09 .2011$ & 32 & 11,94 \\
\hline 17 & $18.08 .2015-25.08 .2015$ & 6 & 10,53 \\
\hline 18 & $29.12 .2015-20.01 .2016$ & 16 & 11,02 \\
\hline 19 & $03.12 .2018-24.12 .2018$ & 15 & 15,62 \\
\hline
\end{tabular}

From the figures below we can see that our parameters start to decrease in crisis states. Such abnormal behavior can serve as indicator or precursor of crashes and critical states.

For the first time, the use of dynamic indicators, precursors of crashes in stock markets using the parameters of a $\alpha$-stable distribution, was proposed by us in the works $[30,31]$ and later repeated in a recent work [32]. Moreover, the authors [32], analyzing only one crisis of 2008 and using a limited set of stock market indices (only three), conclude that the $\beta$ parameter is an even more convincing indicator of the approaching crisis. Our data for a large set of critical events and crashes, as well as stock indices of countries of different levels of development convincingly speak in favor of the $\alpha$ parameter.

Interesting are the conclusions that follow from the analysis of Table 1 . Indeed, the indexes of stock markets, ordered by the value of the $\alpha$ parameter, reveal a characteristic pattern that large $\alpha$ parameters correspond to more advanced stock markets of developed countries. At the same time, the $\beta$ asymmetry parameter also differs markedly from zero. For emerging and emerging markets, the $\alpha$ parameter is noticeably smaller, and the $\beta$ parameter tends to zero.

In our opinion, this indicates that crises in emerging markets occur more often, are more profound and long lasting. This leads to a decrease in the $\alpha$ parameter (see Fig. 3a, c) and leveling of the distribution asymmetry, with the result that the $\beta$ tends to zero.

\section{Conclusions}

Recently, there has been an increasing of interest in the study of quantitative methods for the stability of 
financial objects, especially in crisis situations. It is extremely important to take precisely preventive measures to prevent significant financial losses.

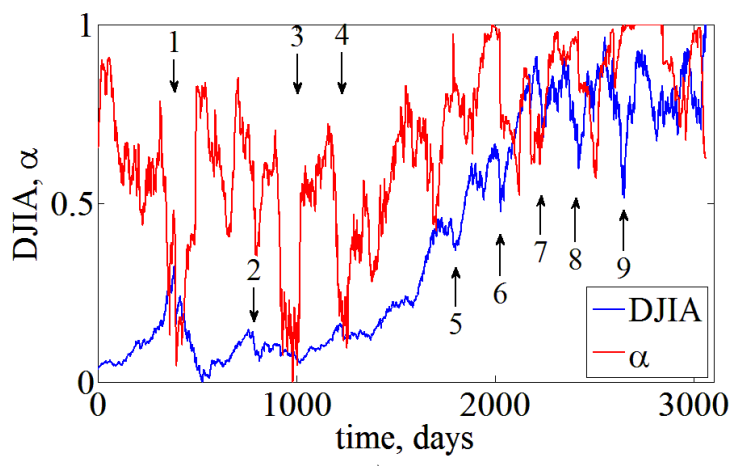

a)

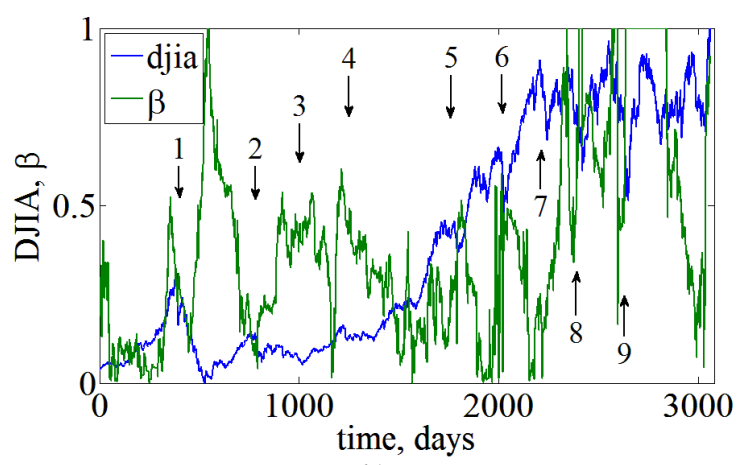

b)

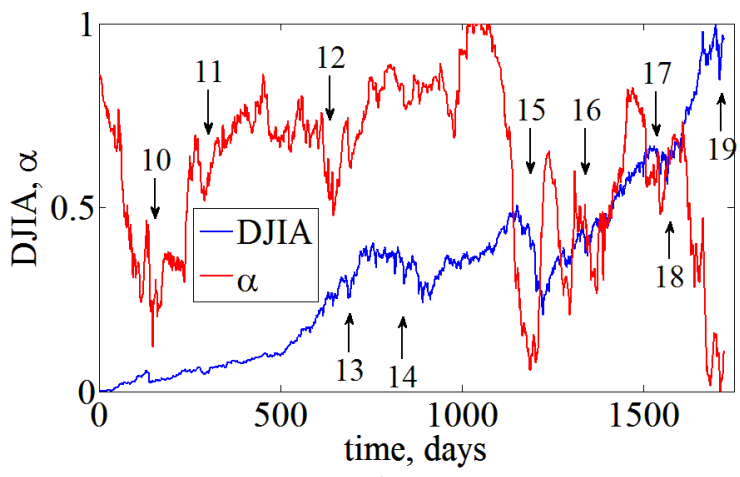

c)

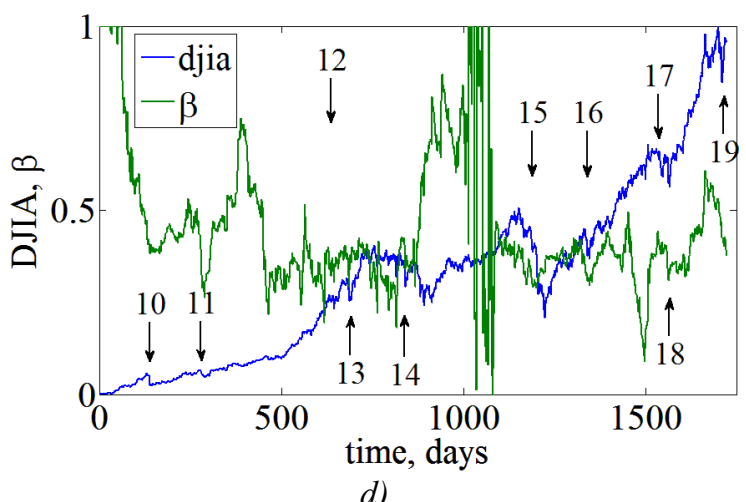

Fig. 3. The corresponding time series and estimated for them parameters $\alpha(a, c)$ and $\beta(b, d)$. Vertical arrows indicate crashes and critical events.
In this respect, an important role is played by the methods of constructing indicators of crisis phenomena, which warn in advance of a possible approaching crisis, that makes them as indicators-precursors of possible crisis states.

Crises manifest themselves in the form of strong price fluctuations of most assets and financial market instruments. In particular, stock market indexes exhibit increased volatility, which is reflected through the appearance of long tails in non-Gaussian probability density functions.

This paper has examined the behaviors of stock markets price fluctuations. As many others results, our research have demonstrated that the fluctuation distribution of DJIA index over the long period of 19002019 are characterized by heavy tails and can be described by the Levy's stable parameters. A similar pattern is observed for other stock indices taken over the shorter period from 2000 to 2019 . Relating on theoretical background of Levy's stable distribution, stock markets time series and normalized log-returns for stock index price, it have been obtained that the Gaussian distribution for stock market is less suitable than Levy's stable distribution.

Further, we have discussed different method for the parameters estimation of the distribution, and pointed out which method is the best. Calculated parameters $(\alpha, \beta)$ have presented a similar behavior for different crisis states and proved that they can be used as indicators of crashes and critical periods. Moreover, it is shown that the absolute values of the distribution parameters themselves characterize the degree of development and efficiency of the stock market itself.

\section{References}

1. Podobnik, B., Valentinčič, A., Horvatić, D., Stanley, H.E.: Asymmetric Lévy flight in financial ratios. Proceedings of the National Academy of Sciences of the United States of America. 108(44), 1788317888 (2011). doi:10.1073/pnas.1113330108

2. Baruník, J., Vácha, L., Vošvrda, M.: Tail behavior of the Central European stock markets during the financial crisis. AUCO Czech Economic Review. 4(3), 281-295 (2010)

3. Bachelier, L.: Théorie de la spéculation. Annales scientifiques de l'École Normale Supérieure, Série 3. 17, 21-86 (1900). doi:10.24033/asens.476

4. Gopikrishnan, P., Plerou, V., Amaral, L.A.N., Meyer, M., Stanley, H.E.: Scaling of the distribution of fluctuations of financial market indices. Physical Review E - Statistical Physics, Plasmas, Fluids, and Related Interdisciplinary Topics. 60(3), 5305-5316 (1999). doi:10.1103/PhysRevE.60.5305

5. Gabaix, X., Gopikrishnan, P., Plerou, V., Stanley, H.E.: A Theory of Power Law Distributions in Financial Market Fluctuations. Nature. 423(6937), 267-270 (2003)

6. Gabaix, X., Gopikrishnan, P., Plerou, V., Stanley, H.E.: Institutional Investors and Stock Market 
Volatility. Quarterly Journal of Economics. 121(2), 461-504 (2006). doi:10.3386/w11722

7. Mandelbrot, B.: The variation of certain speculative prices. The Journal of Business. 36(4), 394-419 (1963). doi:10.1086/294632

8. Levy, P.: Théorie des erreurs. La loi de Gauss et les lois exceptionnelles. Bulletin de la Société Mathématique de France. 52, 49-85 (1924)

9. Gnedenko, B.V., Kolmogorov, A.N.: Limit Distributions for Sums of Independent Random Variables. Addison-Wesley, Cambridge (1954)

10. Fama, E.F.: The Behavior of Stock-Market Prices. The Journal of Business. 38(1), 34-105 (1965).

11. Mantegna, R.N., Stanley, H.E.: Scaling behaviour in the dynamics of an economic index. Nature. 376, 46-49 (1995).

12. Weron, R.: Levy-stable distributions revisited: tail index $>2$ does not exclude the Levy-stable regime. International Journal of Modern Physics C. 12(2), 209-223 (2001).

13. Koutrouvelis, I.A.: Regression-Type Estimation of the Parameters of Stable Laws. Journal of the American Statistical Association. 75(372), 918-928 (1980)

14. Brorsen, B.W., Yang, S.R.: Maximum Likelihood Estimates of Symmetric Stable Distribution Parameters. Communications in Statistics Simulation and Computation. 19(4), 1459-1464 (1990). doi:10.1080/03610919008812928

15. Nolan, J.P.: Maximum likelihood estimation of stable parameters. In: Barndorff-Nielsen, O.E., Mikosch, T., Resnick, S.I. (eds.) Lévy Processes: Theory and Applications, pp. 379-400. Springer Science+Business Media, Boston (2001)

16. Fama, E.F., Roll, R.: Parameter estimates for symmetric stable distributions. Journal of the American Statistical Association. 66(334), 331-338 (1971). doi:10.2307/2283932

17. McCulloch, J.H.: Simple consistent estimators of stable distribution parameters. Communications in Statistics - Simulation and Computation. 15(4), 1109-1136 (1986)

18. Shao, M., Nikias, C. L.: Signal processing with fractional lower order moments: stable processes and their application. Proceedings of the IEEE. 81(7), 986-1010 (1993). doi: 10.1109/5.231338

19. Ma, X., Nikias, C.L.: Joint estimation of time delay and frequency delay in impulsive noise using fractional lower order statistics. IEEE Transactions on Signal Processing. 43(11), 2669-2687 (1996). doi:10.1109/78.542175

20. Nicolas, J.-M., Anfinsen, S. N.: Introduction to second kind statistics: Application of log-moments and log-cumulants to the analysis of radar image distributions. Traitement du Signal. 19(3), 139-167 (2002)

21. Kuruoğlu, E.E.: Density parameter estimation of skewed $\alpha$-stable distributions. IEEE Transactions on
Signal Processing. 49(10), 2192-2201 (2001). doi:10.1109/78.950775

22. DuMouchel, W.H.: On the Asymptotic Normality of the Maximum Likelihood Estimate When Sampling from a Stable Distribution. The Annals of Statistics 1(5), 948-957 (1973)

23. Zolotarev, V.M.: One-dimensional Stable Distributions. American Mathematical Society, Providence (1986)

24. Chambers, J.M., Mallows, C.L., Stuck, B.W.: A Method for Simulating Stable Random Variables: Journal of the American Statistical Association. 71(354), 340-344 (1976).

25. Koutrouvelis, I.A.: An iterative procedure for the estimation of the parameters of stable laws: An iterative procedure for the estimation. Communications in Statistics - Simulation and

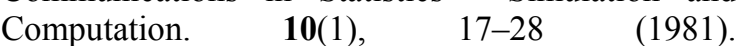
doi:10.1080/03610918108812189

26. Arnold, V.I., Avez, A.: Ergodic problems of classical mechanics. Benjamin, New York (1968). doi:zamm.19700500721

27. Umeno, K.: Ergodic transformations on $\mathrm{R}$ preserving Cauchy laws. Nonlinear Theory and Its Applications. 7(1), 14-20 (2016). doi:10.1587/nolta.7.14

28. Charles, A., Darné, O.: Large shocks in the volatility of the Dow Jones Industrial Average index: 19282013. Journal of Banking \& Finance. 43(C), 188199 (2014). doi:10.1016/j.jbankfin.2014.03.022

29. Duarte, F.B., Tenreiro Machado, J.A., Monteiro Duarte, G.: Dynamics of the Dow Jones and the NASDAQ stock indexes. Nonlinear Dynamics. 61(4), 691-705 (2010). doi:10.1007/s11071-0109680-z

30. Soloviev, V.M., Chabanenko, D.M.: Dynamika parametriv modeli Levi dlia rozpodilu prybutkovostei chasovykh riadiv svitovykh fondovykh indeksiv (Dynamics of parameters of the Levy model for distribution of profitability of time series of world stock indexes). In: Pankratova, E.D. (ed.) Proceedings of 16-th International Conference on System Analysis and Information Technologies (SAIT 2014), Kyiv, Ukraine, May 26-30, 2014. ESC "IASA" NTUU “KPI", Kyiv (2014)

31. Soloviev, V., Solovieva, V., Chabanenko, D.: Dynamics of $\alpha$-stable Levi process parameters for returns distribution of the financial time series. In: Chernyak, O.I., Zakharchenko, P.V. (eds.) Contemporary concepts of forecasting the development of complex socio-economic systems, pp. 257-264. FO-P Tkachuk O.V, Berdyansk (2014)

32. Fukunaga, T., Umeno, K.: Universal Lévy's stable law of stock market and its characterization. https://arxiv.org/pdf/1709.06279 (2018). Accessed 21 Mar 2019 